\title{
LeARNing NOT BURNING (OUT): SUSTAINABLE TIME MANAGEMENT IN ENGINEERING EDUCATION
}

\author{
Nicholas J. Rupar and David S. Strong \\ Queen's University \\ nicholas.rupar@queensu.ca / david.strong@queensu.ca
}

\begin{abstract}
In recent years, there has been some concern raised regarding decreased mental health and wellbeing and increased reports of surface-learning tendencies in Canadian engineering students. Prior papers have noted possible links between these trends and the manner in which students use their time.

Having completed a study on student time use, this paper presents some initial results - including a frequency assessment of reasons for student absences - which seem to support some of the previously hypothesized systemic problems.

Following this, an overview of institution- and instructor-imposed constraints precedes a discussion on how these constraints may be contributing to student time use difficulties, and ultimately to decreased wellbeing and learning capacity.

A sampling of possible interventions are then presented, and important validity considerations for conducting such research are discussed. Research into the efficacy of these interventions, due to lack of existing empirical proof, should be conducted.
\end{abstract}

Keywords: Time Management, Decision Making, SelfRegulated Learning, Self-Efficacy, Interventions, Mental Wellbeing, Academic Time-Based DecisionMaking

\section{INTRODUCTION}

"In second year I remember a prof told us 'your schedules are garbage","

- Student interview participant

Over the past several years, multiple CEEA papers have been presented on topics related to student time management, decision making, and wellbeing. These papers appear to indicate trends towards propensity of surface learning, reduction in class attendance, reduced academic honesty, and increase stress and burnout among Canadian engineering students [1]-[6].

Two prior papers have presented summaries of the literature in this area, which has been called
"Academic Time-Based Decision-Making" (ATBDM) [1],[2]. This paper will build on that research by presenting some initial results from a study on undergraduate engineering ATBDM and then discussing their implications and impacts on student mental health issues and academic capability.

\subsection{Scope, Goals, and Rationale}

When a description of the topic of this paper was mentioned to an engineering professor, they replied "Oh! I am excited to see what students can do to improve their time management!" While individual methods of improving time management from this study will be reported elsewhere, this paper will focus mainly on the institutional factors and remedies.

The reasons for focusing on institutions and instructors are to instill an understanding of the complexity of ATBDM-related problems, to give context to these problems, and to suggest areas and methods of future studies which might determine efficacious solutions. By doing so, the roles of instructors and institutions in both creating and solving these issues will be better understood, allowing for more reliable future studies on student time use, learning efficiency, and/or wellbeing to be conducted and more efficacious recommendations to be made.

\subsection{Background Information}

The term "Academic Time-Based DecisionMaking" was created to encompass both time use and decision making in an academic context [1]. It differs from "time management" (which is not well defined itself [1],[7]) in that time management has tended to focus on individuals' actions, whereas ATBDM considers both individual and systematic forces affecting academic time allocation and use [1].

ATBDM draws on a diversity of theories in motivation, procrastination, decision-making, time management, social time use, and behavioural economics to attempt to explain reported phenomena [1]. Also included in the development of ATBDM 
were analyses of power structures and embedded assumptions in existing time management lore [2]. A prior CEEA paper containing an ATBDM literature review noted that a cultural shift towards hyperindividualism, and a "gig economy" style academia, may be contributing to observed trends [1].

By synthesizing a variety of theories with empirical reports, arguments were made that ATBDM may be linked to topics including mental health and learning efficacy, and that this link could be leveraged to explain negative trends in current Canadian and worldwide engineering education, including reported increases in surface learning tendencies and decreases in student mental wellbeing [1],[2].

\subsection{Methods}

The full research study follows a mixed methods approach. Approximately 200 students from a midsized Canadian engineering school completed a survey (response rate of $\sim 7 \%$ ) on ATBDM topics, after which 10 agreed to complete follow-up interviews. The survey was open from February through April 2020, with interviews conducted in May 2020, after both the exam period and the onset of the Covid-19 pandemic.

Participants who volunteered for a follow-up interview were generally representative of the survey participants, however volunteers exhibiting poorer time management were subsequently less likely to reply and complete the interview when given the opportunity. Survey participants were representative of the population across engineering discipline and year, but tended to have higher academic standing (via self-reported GPA) than would be expected.

Participants were recruited in-class, via email, and via social media in two approximately-equal bursts, one in the week after February break, and the other during the April exam season. The exam season students were asked to respond thinking of a "typical week in the semester" instead of their "last week."

The survey data was collected with the university's institutional survey software, and on average took 15-20 minutes to complete. The interviews on average took 2 hours to complete, and no incentive was given for participation. The data was analyzed using NVivo, SPSS, R, and Excel. Full study methods and methodology are intended to be published at a later date.

\section{StUdent PeRspectives ON STUdent TIME}

Prior papers on ATBDM-related topics have included professor observations and reflections as well as empirical student studies. Some qualitative and some speculative work into reasons for student absences had been completed, as had some reviews on mental health, surface learning, and cheating, all of which were used in the preparation of survey and interview questions [1-6],[8].

From the literature, it appears that engineering faculty and engineering education researchers are aware of issues in these (ATBDM-related) areas, and have some understanding of proximate causes and general trends, especially for mental wellbeing [16],[8]. However, it appears that there is a lack of understanding in the literature of the complexity of interacting causes and of the prevalence of various time-related behaviours (e.g. missing class), especially from a comprehensive student perspective [1]. To help remedy this, this section will present student perspectives on missing classes, on workload and burnout, and on working hours and breaks.

\subsection{Absence, Lateness, and Concentration}

The survey inquired on 11 different reasons for absences or lateness, covering topics from "being too early or late for me" to "sleeping in on purpose," "sleeping in or missing it by accident" to "traffic or weather problems." Only 6 of the $\sim 200$ participants checked "other" suggesting the 11 topics presented comprehensive coverage of reasons for absence and lateness.

The top 5 reasons for being absent from class once or more than once were (with percent of respondents): class "not being an effective method of learning" (72\%), "completing homework" (51\%), "class being too early or too late to be functional" (49\%), "needing a break" (37\%), and "sleeping on purpose" (36\%). In addition, "needing to eat" and "missing a class by accident" were each counted by $28 \%$ of students as a reason to be absent and by $40 \%$ of students for being absent or late.

Overall, the top five reasons for being absent once or more than once affected $84 \%$ of students $(58 \%$ more than once), with an average of 2.4 of the top 5 reasons per student. When including all 11 reasons, $90 \%$ of students were absent for one or more classes (62\% two or more classes), with an average of 3.9 reasons per student. Moreover, $95 \%$ of students were absent or late at least once, with $30 \%$ checking six or more reasons for being absent, and another 33\% checking two to five reasons.

These numbers are astounding: 1 in 7 students missed class because they needed a break more than once in a week. Almost 1 in 2 students missed more than once because they did not feel that the lecture was an effective method of learning. Approximately 1 in 3 students missed more than once because they found the class to be too early or too late for them to function properly. In comparison, only $7 \%$ missed for weather 
or transportation issues once and $1 \%$ more than once, and for work, $2 \%$ once and $2 \%$ more than once. These trends held irrespective of self-reported GPA.

In addition to absences, although $75 \%$ of students said they had never fallen asleep in class in the last week, 1 in 3 said they "very often" or "almost all the time" stopped paying attention in class, a further 1 in 4 said this happened "a few times," and only 1 in 9 said it happened "never."

One theme discovered in the transcripts was that it can be difficult to self-motivate to catch up on missed material. Another theme was the formation of habits of missing classes in many students. In one student's words:

I didn't really skip any classes in the fall semester of second year, I only really started winter semester, I think part of it was that I was so burnt out from all different angles [of things to do] that I just really felt like I needed to sleep so I would miss a lot of my morning classes because I was sleeping until noon - because I would just be so tired ...

It was a lot better this semester: I went to a lot more of my classes. I didn't go to the ones [which] I didn't go to [partly] because it had become a habit to be like "I'm not really feeling it I'll just catch up on it later" um which is not a good idea to do [because] I never really ended up catching up on it ...

I would skip the first two classes in that week or the last two classes and then by the time I went to the next class I would be a little bit behind on the content, like I wouldn't know what is going on but I would just be like its ok I'll just pick up where I am [and catch up later] and then I'd never end up doing it because I had an assignment or a prelab due or whatever so I never really ended up catching up on that content until I had a quiz, and then I would be trying to cram in all this content.

Given such difficulty and regret, it is surprising that so many students find themselves missing classes. Many of the interviewees (except those who claimed to almost never miss a class) also expressed regret at missing classes but often felt caught in a cycle of constantly trying to catch up, which is reflected in the survey results of 1 in 2 students missing to complete homework. This cycle of constantly trying to catch up appears to lead to burnout and other longer-term impacts.

\subsection{Workload, Mental Health, Burnout, and Surface Learning}

Previous reports on the trends of increase in surface-learning and decrease in mental health within engineering students consist of observations, surveys, and interviews [1-6]. The reports indicate that students are alarmingly stressed throughout the academic year, and that they have started requesting more surface-learning approaches, such as only having exam questions that were very similar to practice problem questions [1],[4]. The connection between looming deadlines and increases in surface learning and stress have elsewhere already been established [9].

While the amount of work that students are expected to do may remain the same as in prior years, the quality of time available for completing this work seems to have decreased. A previously presented hypothesis suggests that schedules with many long stretches of class, with several one-hour breaks, and/or with inconsistent day-to-day schedules may decrease students' ability to work efficiently and effectively [1].

Themes of poor schedules leading to poor academic and mental health outcomes were prevalent in the qualitative analyses. One interviewee presents good examples when contrasting two semesters of their own experience: in comparison to their winter semester ( 5 courses), where the student described being in control of their time, having good balance, and feeling able to take weekends off, they describe their fall semester ( 6 courses, dropped to 5 halfway through) as such:

Last semester, that was the most brutal semester I had ever had. There were a lot of one-hour breaks between classes, so it wasn't what I had this semester where I had 3 or 4 hour - like bigger chunks - between [classes] where I actually felt I could do work ...

I went from 8:30 to 5:30 (I had a lot more labs for one) and I would only have 1 hour breaks in between, so I didn't really feel like it was enough time to like get into the rhythm of being productive - it tends to take me an hour to focus in, and then once I'm focused in I tend to be like very [focused] like I don't check my phone, I'm very much getting it done until it gets done ...

These 1 hour breaks, it felt like I really couldn't do anything in them and so I tended to have a lot more stress because I was trying to get everything done those nights, and I stopped working out, which probably didn't make me feel much better, because I wasn't doing exercise, because I was just like "I don't have an hour to give," and I 
would miss dinner a lot because I would be like "I just can't, I don't have time to cook," so I'd order [food] a lot, it was just a lot more trying to fit everything in during that nighttime block instead.

Another theme was cycling into daily and weekly burnouts, with pressure and exhaustion mounting as the day, week, and semester progressed due to lack of perceived opportunity for breaks. Even the most accomplished interviewees (in both academics and non-academics), who seemed to have a workable system, have experienced burnout. As one student said "[burnout] comes and affects everyone eventually."

Perhaps most insidiously, one participant noted that it can be hard to tell sometimes how others are doing: "it's hard to tell if somebody is kind of spinning out or overstressed because it's kind of something that you keep [to yourself] ... you can only really talk about it with your close friends that sort of thing."

\subsection{Working Hours and Breaks}

The theme of unproductive one-hour breaks was also common to most participants. One participant noted that for an hour break, it might take " $\sim 15$ minutes to get mentally prepared and $\sim 15$ minutes at the end to prep for the next class, and 30 minutes in between is not really enough time to do many engineering problems, especially in the upper years." Essentially, there appears to be an overhead cost of 0.5 hours per working break.

Since students tend to have little control (other than to miss class) over their class schedule, clearly there is some onus on the institution to enact appropriate timetabling. Unfortunately, it appears that in many cases, class schedules are characterized by many short breaks or else by many long stretches of lecture without break. One fourth year student expressed it well:

In second year I remember a professor told us 'your schedules are garbage: you have days that go 8:30-5:30 with a half hour break somewhere in there, so please don't skip my class to have lunch, like please just bring in food' or "yeah if you want to skip my class to go to the cafeteria for sure [go do it]"

Our profs were like "bring it (food). I'd much rather you be here eating spaghetti and watching me teach and listening to me than you leave to go do something," so a lot of them were very understanding and open about that.
Several interviewees noted that they did not eat lunch, some because they were typically too stressed to eat during the day and did not have an appetite, and others because they could not find time. One participant noted that they would eat meals despite rules against doing so in certain lecture halls because they had to keep their energy somehow.

Even if one eats in class, it is likely difficult to retain focus for hours on end without a longer break, which may be a contributing factor for the high proportion of survey respondents claiming to often not be focused in class.

\subsection{Deadlines}

Working hours and the feeling of being "always on" relates also to the topic of assignment deadlines. Previous work speculated that late-night deadlines may be causing stress and surface learning because it imposes a strict "end time" (and thus structured time) on what might otherwise be unstructured time [1]. However, in a prior presentation on ATBDM, an audience member (apparently a professor) noted that "students tell us to put the deadlines at midnight, so what are we supposed to do?"

The results of the interviewees did seem initially to support the idea that students prefer late-night deadlines. Several students referenced "midnight" or " $10 \mathrm{pm}$ " deadlines before that line of questioning was established, suggesting that this has become the culturally-embedded idea of when deadlines are "supposed" to be. However, several students (sometimes the same students wanting the midnight deadlines) noted elsewhere that they ideally prefer to go to bed before midnight, sometimes well before midnight.

On further questioning, one student ultimately revealed that the reason for preferring midnight deadlines is primarily because it gives consistency as to when things are due. The theme of inconsistency in deadlines causing additional stress in already hectic daily schedules was prevalent, and subsequent interviewees agreed with consistency being a critical factor. For example, one interviewee noted that they once ran into trouble handing in a report late because they assumed a group project was due at midnight (the deadline in their other classes), not at $10 \mathrm{pm}$.

It appears therefore that students may be requesting midnight deadlines primarily to reduce complexity rather than because it actually benefits their long-term learning or wellbeing. In acquiescing to these requests, it is possible that instructors are collectively, and unintentionally, removing an ideal unstructured learning time - the "evening block."

In addition, if working to a deadline increases stress (positive or negative) and surface learning, it 
may be the case that late deadlines promote more stressful late working times, which in turn may affect student's ability to get up in the morning and go to class. A theme of waking up late or later than expected because of working late the night before - and consequently missing or being late to class - was found throughout interviews and in the survey results.

\section{RECOMMENDED FUTURE AREAS OF STUDY FOR SUSTAINABLE STUDENT ATBDM}

It is important to note that student perspectives on solutions are not necessarily accurate or precise, since they sometimes rely on unproven (and sometimes unexpressed) assumptions. However, many current interventions also seem to rely on poor assumptions, often, for example, that promoting student-based time management or mindfulness training alone will improve student wellness and time allocation. If the appropriate amount and type of time to be allocated is not available (due to poor scheduling or otherwise), these interventions may have a limited ongoing impact.

The data collected in this study is sufficient to describe both problems and probable causes, but is insufficient to support specific solutions. This is a byproduct of the exploratory nature of the study and is complicated by the multi-layered complexity of the uncovered problems.

As such, there is a great need to identify and test hypothetical solutions to see if they do appropriately address the combination of apparent student timepoverty and mental health issues. This section will identify some of the areas which may be studied and intervened from an institutional-level perspective.

It should be noted that these hypothetical solutions are not necessarily optimal, and may themselves cause additional problems. Thus, there remains the need for valid testing, some components of which are outlined in Section 4.

\subsection{Short Overview of Nudge Theory}

Nudge Theory is an empirically-backed economics theory based on behavioural economics, itself a response to the discovery of non-rational economic actors which violated the assumption of strictly rational decision making. "Nudging" is essentially the idea that social structures can be used to influence people towards a default result, without being overly restrictive, under the assumption that people will tend to choose the default option when they have no reason to choose otherwise. [10]

A basic engineering-style example of this principle is creating a sidewalk for pedestrians: a sidewalk does not preclude people from walking on a road, but it suggests an area of least-resistance to be used in most cases which subsequently has the benefit of reducing potential collisions between pedestrians and cars.

Some of the suggestions below attempt to use Nudges to combat existing (negative) default directions, and redirect students (relatively) easily to a more optimal path. It must be noted that in many cases this is not making it "easier" for students so much as it is removing accidentally-imposed hardships due to a disregard for negative externalities in prior solutions.

\subsection{Faculty-Wide "Protected Times"}

The first proposal is to have faculty-wide recommendations (or regulations) on when deadlines can occur during a week. This is not an entirely new concept, as current bans on deadlines during Reading Weeks and pre-exam breaks do exist (even if they are not always respected).

Such protected times would nominally target evenings and weekends, but the exact times must still be discovered. Theoretically, based on interview responses, an evening deadline should definitely be no later than $10 \mathrm{pm}$, although 9:30 pm or earlier may be more optimal.

For weekends, one theme from the interviews was that students would take one of the two weekend days off, either on purpose or because of exhaustion. Thus, it is likely that at least one day without deadlines would be ideal, although it is unknown which day would be best, or whether both would be ideal. It may also be the case, for example, that weekend-evening deadlines should be earlier than weekday-evenings, although this too will have to be evaluated.

It may also be beneficial to suggest protected times in which communication is limited to students (e.g. from online learning modules as well as instructors emails), however this may be more difficult to deploy and may or may not produce as much benefit - no theme was encountered in this study where dealing with communications was seen as a hardship (although it may still be an unacknowledged stressor).

\subsection{Pre-Scheduled Meal Breaks}

There are many benefits to having rolling meal breaks from a macro-efficiency perspective: for example, if everyone on campus had a break from 12$1 \mathrm{pm}$, eating establishments would be overwhelmed with an influx of patrons and such a surge capacity may not be feasible. However, it is apparent from the reported interview and survey results that students might benefit from a suggested break around the midpoint of their day, that currently it can be difficult 
to ensure this break occurs without missing class, and that some students are routinely not eating lunch due to being overly stressed.

Scheduling suggested meal-breaks directly into timetables may be one solution to this problem. If scheduled as a "class," such a break might be relatively easily integrated into an existing timetabling process. Having the break time in an official timetable would serve as a "nudge" for students to take that break. Nominally, lunch breaks are recognized as important in a workplace, so why not also in a university setting?

In addition, investigating whether a 1.5-hour dinner break (e.g. 5:30-7:00) may be more beneficial than a 1-hour dinner break (or no official dinner break) may have merit. This concept originated with one interviewee who noted that they tended to take approximately 1.5 hours for dinner, downtime, and reuptake when given the opportunity. When not going to an evening class, this time length seemed reasonably consistent across interviewees. Like lunches, it may also be useful to program dinner breaks into timetables directly.

The trade-off, however, would be having classes go until $10 \mathrm{pm}$, which may be problematic from a sleep perspective. Anecdotally, the difference of 9:30 versus 10:00 pm may be discovered to be insignificant - i.e. the half-hour of break may be more efficiently placed with dinner. It may be the case that some engineering schools already provide such a length, in which case a comparative study might be conducted.

Ironically, at the beginning of the pandemic, it seems that several workplaces, including at least one university engineering department, suggested that their staff and faculty keep a regular lunch time, end the workday at a consistent and reasonable time, and take small breaks throughout the day.

\section{4. "Not Enough Time and Space": the Opportunity to Blend Live and Pre- Recorded Lectures}

Due to increasing enrolment and insufficient classroom space, the authors' home institution officially extended "regular" daytime class hours from $5: 30 \mathrm{pm}$ to $6: 30 \mathrm{pm}$ in 2019 , with some core courses scheduled even later. In a number of cases, students have no dinner break whatsoever.

Scheduling conflicts due to room constraints also may be the cause of both multiple 1-hour breaks and of 6-hour lecture marathons. Fortunately, there may be a method which allows for reclamation of this time and control: pre-recorded lectures and/or virtual live lecture spaces.

Across the interviewees, there seemed (at the start of the pandemic) to be broad support for returning to in-person lecturing post-pandemic. Some, however, pointed out the advantages of being able to pause and re-play pre-recorded videos - a different type of advantage than asking live questions. Some combination of pre-recorded and live lecture methods may therefore free up lecture hall space and allow for more optimal organization of schedules while maintaining instructor contact time.

Prior to the pandemic, some courses were structured with the first lecture of the week offered online and the following two hours of lecture offered in-person. A disadvantage of this, pointed out in the interviews, was that it is suspected that some students do not watch the videos before coming to class. However, if many students appear to be missing class regardless - and as several interviewees noted do not usually catch up on that material despite best intentions - then perhaps one video lecture per week is the "lesser of two evils".

In the interviews, there was some agreement that no more than $1 / 3$ of lecture time in a week should be pre-recorded, although ideally a controlled trial would set the optimal proportion (note that surveys asking simply "how many lecture hours would you want in person versus pre-recorded per week" may not be a reliable method to poll students' opinions on this, especially pre-experience). Just as with the breaks, nudge theory suggests that placing these virtual lecture times into a timetable may better suggest to students when to view those videos, which in turn may support beneficial habits and result in higher completion rates.

\subsection{Re-evaluation of Evening Midterms}

It was noted by at least two students that one evening midterm per week was reasonable, but two evening midterms would begin to place abnormal stresses on their week. Because of the apparent tight margins on student work time - potentially exacerbated by some imposed low-quality times described above - it was noted that these perturbations have cascading effects into the next week and beyond.

Evening midterms have been implemented for a variety of convenience reasons for both instructors and students. For example, an evening midterm allows for coordinated times among various sections, provides more time than available in a 1-hour class (which also helps with accommodations), and reduces the rush to transfer to and from other classes before and after the midterm. However, evening midterms can also interfere with other evening classes or consume a valuable, uninterrupted work or recuperation period.

As such, it is recommended that a study into the trade-offs of evening midterms be conducted, and that that study report on both proximate and ongoing consequences (both positive and negative). The study should also attempt to determine the optimal and 
maximum number of evening midterms per week and/or per midterm period.

It is possible that mandatory evening classes (as opposed to elective evening classes) may be causing stress as well because they are "imposed" rather than "selected." There is some evidence from the interviews to support this notion, especially with multiple evening classes in a week, however those particular interviewees also had a higher-than-usual course load, so this conclusion is unreliable.

\section{METHODS TO IMPROVE THE VALIDITY AND RELIABILITY OF FUTURE STUDIES}

One of the important findings from the research study was the complexity required to understand the social aspects of time and timely interactions. Different types of time and time management strategies can affect the probing of time use itself, as can differences in perception and positions of power. As such, some considerations are provided here for future studies on ATBDM, including engineering student time use.

\subsection{Negative Externalities and Paradoxes of Efficiency}

Negative externalities in economics are unintended and unaccounted for consequences of particular actions [10]. An effect can be considered a negative externality when the entity responsible for creating it does not directly pay for its consequences. In an academic time-based setting, this may be, for example, when a course schedules a midterm at the same time as another lecture, or when an institution schedules classes over a traditional break period.

Paradoxes of efficiency result when a portion of a system is made efficient at the cost of reducing overall system efficiency [11]. Examples in academic time use would be when a student hurries to finish a piece of homework for a deadline without internalizing the information they are meant to learn.

Both of these fallacies must be considered when exploring time use because of the limited amount of time available to students - namely 24 hours per day and/or 12 weeks per academic semester. Understanding the lifecycle impact of any resource is required for sustainability, and time is no exception.

\subsection{Types of Time and Variety of Strategies}

There are at least seven different types of time experienced by students at university [1]. These range from strict, externally-scheduled time to periods of uninterrupted work time to opportunistically found time. Understanding that one hour is not necessarily the same as any other hour is important when trying to quantitatively compare hours spent on a course. For example, a student may spend three 1-hour breaks on a subject and report " 3 hours" of indeterminate-quality work but only functionally complete 1.5 hours of poorquality work, without consciously being aware of it.

Similarly, conclusions from the Pilot study and initial data analysis of this study seem to suggest that a variety of time management techniques may be successfully employed by students [2]. Assuming a single style or technique as being useful for everyone may be problematic from an efficiency and a selfefficacy perspective, since students may get frustrated and have a reduced working efficiency while continuously trying to find techniques that work for them. This may be especially true if a given technique is lauded as being universally useful.

\subsection{Delayed Onsets and Cause-and-Effect}

It was noted several times by interview participants that, in the struggle to catch up on material, "bad days" can compound and "bad weeks" spill over into the following weeks. Understanding that any negative results from a "bad day" may not become apparent immediately is important to prevent false-negative study results. This is especially true in assessing for long-term efficacy of interventions designed to reduce stress and burnout.

It is also possible that direct causes and effects are difficult to ascertain given the complexity of the problems. As exemplified above by the requests for midnight deadlines, the ultimate cause is sometimes not immediately apparent to researcher nor participant. Simple ATBDM solutions should be initially treated with skepticism due to the apparent propensity to oversimplify notions around time.

\subsection{Sampling Biases and Trustworthiness}

The process of recruitment for this study appeared to bias slightly towards higher-self-reportedGPA students in the survey and better time-managing students in the interviews. Understanding that it takes time and effort to answer ATBDM questionnaires was a focal point in deciding recruitment methods and survey size for this study, and should be a significant consideration for subsequent studies as well.

Trustworthiness of data is also sometimes difficult to assess, as students already pressed for time or under stress may inadvertently over-simplify answers, give expected answers, or give cultural-level answers instead of personal answers. In this case also, midnight deadlines being the assumed default is a good example. Care should be taken in interpreting future results to understand the context of answers. 


\section{Ethical Trade-OfFs in Potential SOLUTIONS}

Future recommendations coming from specific studies should include balancing the costs and benefits of different strategies from several perspectives. Some dilemmas that may arise might include how much to push students to find their limit, and in what manners should they be pushed? Should policies, for example setting deadline guidelines, favour traditionally good students (reward) or help lower-achieving students (support). What is the balance of responsibility and effort among student, instructor, and institution in instituting a time-friendly working environment?

The most important consideration here is that these trade-offs in accountability be explicitly named and considered, or at least explicitly assumed, so that responsibilities and duties are clearly outlined.

\section{CONCLUSIONS}

Sustainable learning practices in engineering education must be based on sustainable time use and decision making principles. Prior papers have noted that, recently, the focus of student time use has often been the allocation of time by students, and not the allocation of time to students by institutions. Compounding assumptions regarding the fungibility of time and the time use preferences by student, faculty, and institutions appears to be contributing to the degradation of student mental health and wellbeing as well as student learning capacity.

Due to the complex, compounding nature of these problems, there are several practices that must be further investigated to establish their full lifecycle impact on student wellbeing and academic capacity. Examples of these include optimal deadline times to reduce strain and complexity, default meal-break times to nudge students to healthy habits (and protect students from potentially harmful intrusions into those times), and blending pre-recorded and live lectures to better allocate working and break times.

There is a pressing need for researchers in engineering education to study these issues and potential interventions. When conducting such research, it is essential to consider the various paradoxes and fallacies associated with complex problems as well as remove faulty assumptions regarding the nature of time, including specifically the assumption that all hours are interchangeable.

The recommendations sections of future studies on time-based interventions should also include a discussion on the ethical trade-offs considered when making time-based recommendations, including by clearly noting who holds the responsibility for control over various time-packets and clearly listing the benefits and costs to different stakeholders (including students, instructors, and institutions).

\section{ACKNOWLEDGEMENTS}

The authors would like to thank all of the engineering student participants who took the time to complete the survey, and especially those who took the time to participate in the interviews. The authors would also like to thank the Faculty of Engineering and Applied Science at Queen's University for their financial support in this research.

All procedures performed in this study involving human participants were in accordance with the ethical standards of the Queen's University General Research Ethics Board per 6027685.

\section{REFERENCES}

[1] N. J. Rupar and D. S. Strong, "What to do? A Review of the Academic Time-Based Decision-Making Literature," Proc. 2019 Canadian Engineering Education Association (CEEA19) Conf., 2019.

[2] N. J. Rupar and D. S. Strong, "Time for a Change? A Research Update and Pilot Study Results on Academic Time-Based Decision- Making," Proceedings of the Canadian Engineering Education Association (CEEA), Jun. 2020, doi: 10.24908/pceea.vi0.14173.

[3] R. Brennan, "A Systematic Review of Canadian Engineering Education Research 2004-2017," 1, Dec. 2018, doi: $10.24908 /$ pceea.v0i0.13079.

[4] R. Clemmer, K. Gordon, and J. Vale, "Will That Be on the Exam? - Engineering Student Perceptions of Assessments, Memorization, and Approaches to Learning," Proc. 2018 Canadian Engineering Education Association (CEEA-ACEG18) Conf., 2018.

[5] S. Doré, "What Seniors Have to Say About Their Engagement," Proceedings of the Canadian Engineering Education Association (CEEA), Jan. 2017, doi: $10.24908 /$ pceea.v0i0.6475.

[6] D. M. Smith and S. Maw, "Supplementary Results of the CAIS-1 Survey on Cheating in Undergraduate Engineering Programs in Saskatchewan," Proceedings of the Canadian Engineering Education Association (CEEA), Mar. 2018, doi: 10.24908/pceea.v0i0.10578.

[7] B. Aeon and H. Aguinis, "It's About Time: New Perspectives and Insights on Time Management," Acad. Manag. Perspect., vol. 31, no. 4, pp. 309- [15] 330, Nov. 2017.

[8] N. Manjikian, "Reflections on Five Years of Coherent Teaching Accross Three Courses on Digital Logic and Computer Systems," Proc 2017 Can. Eng. Educ. Assoc. CEEA17 Conf, 2017.

[9] R. McCord and H. Matusovich, "Study Context Matters: A Case Study on How Time Crunches Lead to Coping Modes of Learning," presented at the 2017 ASEE Annual Conference \& Exposition, Columbus, Ohio, Jun. 2017, doi: 10.18260/1-2-28875.

[10] Kahneman, Daniel. Thinking, Fast and Slow. 2015.

[11] Tenner, Edward. (2019, April). "The paradox of efficiency" [video]. TED Conferences.

https://www.ted.com/talks/edward_tenner_the_paradox _of_efficiency?language $=$ en 